\section{Hirnmetastasen: Der wahre Nutzen der SRS}

\begin{abstract}
Die stereotaktische Radiochirurgie (SRS) läuft der Ganzhirnbestrahlung (WBRT) bei der Behandlung von Hirnmetastasen immer mehr den Rang ab, solange die Zahl der Metastasen eines Patienten überschaubar ist. Aber ist die gezielte Bestrahlung der Herde wirklich die bessere Wahl?
\end{abstract}

$B^{\text {is }}$ eiben Hirnmetastasen unbehandelt, ist die Prognose sehr ungünstig - mit Überlebenszeiten von 1-2 Monaten. Die seit Mitte des vergangenen Jahrhunderts angewendete WBRT konnte sie immerhin auf 4-6 Monate verlängern und wurde schnell zum Standard. Doch in jüngerer Zeit greift man zunehmend auf eine neue Technik zurück, die SRS. Dabei werden stark gebündelte Strahlen auf ein präzise definiertes Ziel gebracht. Das hat Vor- und Nachteile: Das umgebende Gewebe wird zwar weitgehend geschont, aber die SRS erreicht somit auch nur die mittels Bildgebung bereits entdeckten Metastasen. Die WBRT trifft dagegen auch mikroskopisch kleine Herde im ganzen Gehirn. Zu ihren Vorteilen gehört nicht nur das Unterbinden zusätz- licher Hirnmetastasen, sie ist auch kostengünstiger und leichter verfügbar. Die SRS geht dagegen mit weniger neurokognitiven Nebenwirkungen und einer kürzeren Behandlungsdauer einher.

Retrospektiv verglichen Lia M. Halasz und Kollegen deshalb die Wirkung beider Verfahren auf das Gesamtüberleben (OS). An 5 Institutionen wurde das OS der Patienten erfasst, deren Hirnmetastasen mit SRS bzw. WBRT bestrahlt wurden. Primärtumor war entweder ein nichtkleinzelliges Lungenkarzinom (NSCLC) oder ein Mammakarzinom gewesen. Die Zahl der Metastasen, das Ausmaß extrakranieller Metastasen und das Behandlungszentrum wurden mittels Propensity-Score-Matching berücksichtigt.
Eine SRS der Hirnmetastasen hatten $27,8 \%$ der Patienten mit NSCLC und 13,4\% der Mammakarzinompatientinnen erhalten. Darunter waren nur wenige Patienten mit mehr als 3 Hirnmetastasen oder Läsionen von $>4 \mathrm{~cm}$ Größe. Patienten mit weniger als 4 Hirnmetastasen, die $<4 \mathrm{~cm}$ groß waren (NSCLC: $\mathrm{n}=189$; Mammakarzinom: $\mathrm{n}=117$ ), lebten länger mit SRS als mit WBRT. Die Hazard Ratio beim NSCLC lag hier bei $0,58(\mathrm{p}<0,01)$, beim Mammakarzinom bei $0,54(\mathrm{p}<0,02)$.

Fazit: Liegen bei NSCLC oder Mammakarzinom weniger als 4 Hirnmetastasen mit einem Durchmesser von höchstens $4 \mathrm{~cm}$ vor, führt eine SRS zu einem längeren Überleben als eine WBRT. Dies gilt auch, wenn Störvariablen, die zur Selektion eines der Verfahren führen, herausgerechnet werden.

Christina Berndt

Halasz LM et al. Comparative Effectiveness of Stereotactic Radiosurgery Versus Whole-Brain Radiation Therapy for Patients With Brain Metastases From Breast or Non-Small Cell Lung Cancer. Cancer. 2016;122(13):2091-100.

\title{
Kopf- und Halstumoren: Beobachten statt Neck-Dissection
}

Bei Patienten mit fortgeschrittenem Plattenepithelkarzinom im Kopf- und Halsbereich (SCCHN) sind oft auch die Lymphknoten befallen. Neueren Daten zufolge scheint ein vollständiges Ausräumen der Lymphknoten ohne konkreten Befund aber nicht sinnvoll.

$B^{c}$ ei kompletter Remission nach Chemoradiotherapie von Kopf-Hals-Tumoren beträgt das Rezidivrisiko unter $10 \%$. Es könnte also sinnvoller sein, eine Neck-Dissection nur bei besonders gefährdeten Patienten vorzunehmen und diese mittels Bildgebung zu identifizieren. Schließlich geht die Ausräumung aller Lymphknoten häufig mit Komplikationen einher. Diese Strategie wurde prospektiv randomisiert untersucht.

564 SCCHN-Patienten mit N2- oder N3-Status und ohne Fernmetastasen (M0) erhielten randomisiert entweder obligat eine Neck-Dissection oder lediglich dann, wenn 12 Wochen nach Ende der Chemoradiotherapie in der Bildgebung mittels kombinierter Positronen-
emissions-/Computertomografie (PET/ CT) Hinweise auf ein Residuum vorlagen. Primärer Endpunkt war das Gesamtüberleben (OS). $17 \%$ der Patienten hatten ein N2a-, $62 \%$ ein N2b-Stadium, $84 \%$ ein Oropharynxkarzinom, $75 \%$ eine p16-Expression im Tumor als Hinweis auf humane Papillomviren. Das mediane Follow-up betrug 36 Monate.

In der Gruppe mit PET/CT-Überwachung wurde seltener eine Neck-Dissection durchgeführt als in der Gruppe mit obligatem Eingriff (54 vs. 221). Chirurgische Komplikationen traten aber in beiden Gruppen vergleichbar häufig auf (42 und $38 \%)$. Die Rate für das 2-Jahres-OS betrug $84,9 \%$ in der Überwachungs- und $81,5 \%$ in der Gruppe mit geplanter Ope- ration. Die Hazard Ratio für Tod fiel leicht zugunsten der PET/CT-Gruppe aus. Keinen signifikanten Einfluss hatte die p16-Expression. Die Lebensqualität war in beiden Gruppen ähnlich. Die PET/CTgeführte Überwachung führte im Vergleich zur Neck-Dissection zu Ersparnissen in Höhe von 2.200 US-Dollar pro Patient über die Dauer der Studie.

Fazit: Patienten mit fortgeschrittenem Plattenepithelkarzinom, bei denen nach der Chemoradiotherapie eine Lymphknotenausräumung an einen positiven PET/CT-Scan gebunden ist, leben genauso lange wie Patienten mit geplanter vollständiger Neck-Dissection. Die Strategie der PET/CT-Überwachung führte aber $\mathrm{zu}$ erheblich weniger Operationen und war kosteneffektiver. Christina Berndt

Mehanna H et al. PET-CT Surveillance versus Neck Dissection in Advanced Head and Neck Cancer. N Engl J Med. 2016;374(15):1444-54. 